\title{
EXAMEN FILOSÓFICO DEL VOCABULARIO SOCIOLÓGICO
}

\section{Introduccion}

Quien se acerca a las ciencias sociales desde las ciencias naturales se siente inicialmente repelido por la oscuridad de la jerga, la pobreza e inexactitud de las ideas, y las pretensiones de hacer pasar la búsqueda de datos sin importancia por investigación científica, y la doctrina imprecisa por teoría científica. Por ejemplo, el eminente biólogo de Princeton, John Tyler Bonner, escribe que la sociología humana "pareće una ciénaga, una mezcla lóbrega de lo obvio y lo oscuro". Esta impresión negativa es justificada sólo a medias. Es verdad que la sociología es una ciencia subdesarrollada, pero también es cierto que se ha lanzado finalmente por el buen camino. Por "buen camino" entiendo el camino de la ciencia.

No ha sido fácil llevar a las ciencias sociales por el camino de la ciencia ni les es fácil permanecer en él. En primer lugar porque han nacido de las humanidades y de la ideología, en segundo porque siguen sometidas a presiones de ambos lados. No es que el contacto con las humanidades y con la ideología sea pernicioso en sí. Tales contactos son inevitables y pueden ser beneficiosos. Lo que es pernicioso para las ciencias sociales, y para cualesquiera otras, es someterse al control unilateral de ideas incorrectas, sean filosóficas, sean ideológicas. Me explicaré.

Las ciencias sociales, y en menor medida todas las demás ciencias, están ubicadas entre la filosofía y la ideología. La filosofía se cuela en las ciencias por el andamiaje general o visión del mundo, por los problemas, por los métodos, por los fines y por la manera de evaluar unos y otros así como los resultados de la investigación. Si la filosofía es rigurosa y está al día con la ciencia, su influencia sobre la ciencia será positiva: la ayudará a aclarar, depurar y sistematizar sus ideas y procedimientos. Pero si la filosofía es dogmática, si se limita a repetir y comentar en lugar de proponerse problemas nuevos y de adoptar técnicas nuevas, entonces su influencia sobre la ciencia será nefasta, ya que le impedirá avanzar.

En cuanto a las ideologías sociopolíticas, es natural que influyan sobre las ciencias sociales. Al fin y al cabo, las ideologias de ese tipo se ocupan de problemas sociales: de ponerlos de manifiesto o de ocultarlos, según el caso, y de promover su solución o de obstaculizar todo esfuerzo por resolverlos. La cuestión no es entonces apartar a la sociología de la ideología y conservarla virgen para que llene las revistas y libros de datos y de ideas que no tengan influencia sobre la sociedad. La cuestión es más bien aparear a la sociología 
con una ideología ilustrada y al servicio del pueblo, una ideología que, lejos de imponer soluciones prefabricadas a problemas no estudiados, acepte que los problemas sociales deben estudiarse antes de interferir con ellos. No se trata de postergar la solución de problemas apremiantes sino de encararlos cientificamente para evitar que empeoren y para evitar el fracaso de una acción bien intencionada pero improvisada y por tanto ciega. En suma, no se trata de impedir el casamiento de la sociología con la ideología sino de promover una unión fértil y útil a la sociedad.

Cuando se piensa en los problemas sociales contemporáneos no se puede menos que espantarse. El Tercer Mundo, en particular, está plagado de problemas espantosos y que, en lugar de ir resolviéndose, empeoran día a día. Baste mencionar el hambre, la sobrepoblación, la desocupación, la marginación, la explotación irracional de los recursos naturales, la dependencia, el bajo nivel educacional, la cultura raquítica e imitativa, la tiranía política, la corrupción, y el militarismo. Cuando el científico acostumbrado a una metodología rigurosa piensa en estos problemas, no tiene derecho a adoptar la actitud fácil de menospreciar o compadecer a los sociólogos empeñados en estudiarlos, ni a los políticos y estadistas que dicen querer resolverlos. La actitud constructiva es poner el hombro, sea convirtiéndose él mismo en científico social ocasional, sea promoviendo la transferencia de metodología de las ciencias maduras a las inmaduras, en este caso las sociales.

Cualquiera sea el modo de colaboración que elija el científico proveniente de una ciencia madura, es probable que proponga un saneamiento del vocabulario sociológico, vocabulario en gran medida impreciso que no se presta al pensamiento riguroso ni a la comunicación clara. Por supuesto, el problema del saneamiento del vocabulario sociológico no se resuelve con sólo escribir un nuevo diccionario de ciencias sociales, sino elaborando teorías exactas, profundas y comprobables acerca de hechos sociales. Pero esta otra tarea, que es la más urgente en el momento actual, puede ser facilitada por una crítica de las aberraciones verbales, ya que éstas no son sino manifestaciones de la pobreza teórica que se quiere corregir.

El presente trabajo es una contribución a la faena de limpiar el vocabulario de la sociologia. Fue inspirado en la participación que le cupo al autor en una investigación de la CEPAL, dirigida por el profesor Aldo Solari, acerca de los estudios sobre dependencia y subdesarrollo de América Latina. Puesto que no tiene otro orden que el alfabético, puede leerse al azar.

\section{Dependencta}

Parece admitido que Argentina es un pats dependiente, al par que Canadá es independiente. Las nociones en juego en este caso son dicotómicas, o sea, de la clase blanco-o-negro. De hecho hay grados de dependencia. Por 
ejemplo Argentina, aunque dependiente en muchos aspectos, no lo es en todos, y lo es menos que Paraguay. Es obvio entonces que necesitamos un concepto más refinado: es preciso hablar de dependencia en ciertos respectos y en determinados grados. Un análisis de la situación hecha en términos de estos conceptos daría lugar a una descripción más exacta y acaso abriría camino a teorías propiamente dichas (no ya meras opiniones ni doctrinas) sobre dependencia. (Ver Teoria.)

Los conceptos más refinados que se necesitan pueden obtenerse de la manera siguiente. Se parte de la expresión intuitiva:

$w$ depende de $x$ en el respecto $y$ en grado $z$, que se simboliza Dwxyz. Luego se fijan algunas de estas variables y se estudian las relaciones binarias y ternarias que resultan o bien se adopta un camino más ambicioso: el de la cuantificación. Esto es, se reemplaza la relación por una función. Por ejemplo, un posible indice de dependencia económica (o dependencia en el respecto $e$ ) de un país $w$ para con un país $x$ sería

$D(w, x, e)=z=\frac{\text { Volumen de la producción de } w \text { contralada por } x}{\text { Volumen de la producción total de } w}$.

(Se puede incluso pensar en una función para cada tipo de producto.) Dando valores a $w$ (o sea, tomando diversos países dependientes) se obtienen distintos grados de dependencia económica, o sea, distintos valores de $z$, v. gr.

$$
D\left(w_{1}, x, e\right)=z_{1} \quad, \quad D\left(w_{2}, x, e\right)=z_{2}
$$

donde $z_{1}$ y $z_{2}$, ambas fracciones, miden el grado de dependencia (en el respecto $e$ ) de los paises $w_{1}$ y $w_{2}$ respecto del país $x$. (Para lograr un análisis más fino se sustituirán países por regiones: de este modo se dará cuenta del colonialismo interno.)

Una vez construido un concepto cuantitativo de dependencia (en distintos respectos) se podrá definir sin dificultad el concepto comparativo empleado al comienzo de este parágrafo. Por ejemplo, se dirá que $w_{1}$ es más dependiente de $x$ que $w_{2}$, en el respecto $e$, si y sólo si $z_{1}>z_{2}$. Y dando valores a la variable $x$, que representa a las metrópolis, se obtendrá, por ejemplo para un mismo pais dependiente $w$ :

$$
D\left(w, x_{1} e\right)=z_{1}^{\prime} . \quad, \quad D\left(w, x_{2}, e\right)=z_{2}^{\prime}
$$

Diremos que $w$ depende más de $x_{1}$ que de $x_{2}$, en el respecto $e$, si $z_{1}^{\prime}>z_{2}^{\prime}$. 


\section{Causalidad y posibilidad}

Muchos filósofos piensan que es menester reemplazar la causalidad por la posibilidad. Alguños cientificos han adoptado esta tesis. Así, p. ej., Kalman Silvert sostiene que en las ciencias sociales es más adecuado hablar de acontecimientos o de circunstancias que hacen posibles otros hechos, en lugar de decir que los causan. Sin embargo, tal sustitución no siempre es posible y, en todo caso, la distinción no se limita a las ciencias sociales. La diferencia se manifiesta en todas las ciencias y rara vez es posible reemplazar un concepto por el otro. Una condición que posibilita un hecho suele llamarse condición necesaria; si se dan todas las condiciones necesarias y suficientes para que se produzca un hecho, suele hablarse de causación. Por ejemplo, un elevado nivel de alfabetización es condición necesaria, aunque no suficiente (no es causa), para la implantación de una industria que emplea una tecnología avanzada; otras condiciones son la existencia de capitales y de mercado, así como de una empresa. Por sí sola ninguna de estas condiciones explica la aparición de la industria en cuestión. Análogamente, el que en un país se den las condiciones necesarias para una revolución (económica, cultural o meramente política) no implica que tal revolución se produzca. En resumen, es preciso retener la distinción entre posibilidad y necesidad causal.

Aun si se logra exactificar una noción de posibilidad, esto es, si se introduce el concepto de probabilidad, es menester distinguir entre posibilidad y causación. Por ejemplo, la probabilidad de que un organismo muera alguna vez es igual a la unidad, de lo cual no se sigue que el nacimiento sea la causa de la muerte. Otro ejemplo: la movilidad social puede tratarse como una cadena de Markov. Pero aun disponiéndose de la matriz de transición (entre grupos sociales) no se dice cuáles son las causas de las diversas transiciones. En otras palabras, un mero enunciado de posibilidad, aun cuando se trate de posibilidad cuantificada, no basta para explicar. La causalidad no ha desaparecido de las teorías estocásticas sino que es un aspecto subordinado de ellas. Por ejemplo, en la mecánica estadística se conservan las fuerzas que causan movimientos así como los choques que desvían a las partículas de sus trayectorias; ambos conceptos se conservan en la mecánica cuántica, otra teoría probabilista. En estas teorfas se puede calcular la probabilidad de que una causa produzca determinado efecto antes que otro: se conservan los conceptos de causa y efecto aunque no el de ley causal que los enlaza.

\section{Decisión}

Los sociólogos influidos por la escuela histórico-cultural suelen afirmar que las ciencias sociales no pueden ser exactas porque tratan con finalidades, valoraciones y actos deliberados, todo la cual trasciende a la naturaleza y es 
rebelde a la matematización. Que se trate de objetos culturales antes que naturales, es verdad. Que no sean matematizables es falso, y lo ha sido desde el momento en que nació la teoría de la decisión, hace de esto ya dos siglos. Esta teoría estudia matemáticamente las decisiones que se toman en vista de alcanzar determinados fines (objetos valiosos) con ayuda de ciertos medios Esta teoría rompe la barrera hecho-valor, pues trata a la vez de medios (a los que asigna probabilidades de conducir a los fines considerados) y fines (a los que asigna valores o utilidades). Y, puesto que todo acto deliberado es precedido de una decisión razonada, no puede decirse ya que las ciencias sociales sean forzosamente inexactas por ocuparse de actos deliberados cometidos con el fin de realizar finalidades valiosas. La teoría de la decisión se ha incorporado ya a la sociología de avanzada, en particular a la politología.

Los autores que optan por el colectivismo metodológico (p. ej. Parsons) no tienen inconveniente en hablar de las decisiones tomadas por un grupo social. En rigor ésta es una mera metáfora: una decisión es un hecho que puede ocurrir solamente en un cerebro. Ciertamente, los miembros de un grupo pueden ponerse de acuerdo, pero esto no quiere decir que el grupo, como tal, es decir como ente supraindividual, sea capaz de tomar decisiones. Lo que sucede en este caso es que cada uno de los individuos componentes del grupo toma la misma decisión que los demás y, más aún, decide que obrará de concierto con ellos. Ciertamente, se puede hablar elipticamente de una decisión de grupo, o tomada por un grupo. Pero no es esto lo que quieren los pensadores influidos por el totalismo de Hegel y la psicología de la Gestalt, quienes (al igual que el hombre primitivo) proyectan sus propios estados psíquicos al mundo exterior.

\section{Definiciones operacionales e indicadores}

Los sociólogos y psicólogos han tomado de los físicos la infortunada expresión "definición operacional". No hay tal cosa. Toda definición es una operación estrictamente conceptual consistente en identificar dos conceptos, uno de los cuales (el definidor o definiens) ha sido introducido o aclarado con anterioridad.

Lo que a menudo se llama "definición operacional" no es ni definición ni operacional, sino simplemente una relación entre variables inobservables, por una parte, y variables observables o medibles, por la otra. Las segundas actúan como indicadores o indices de las primeras. Así se dice, por ejemplo, que los niveles de la bolsa de valores constituyen un índice o medida (ambigua) del estado de la economía de un país capitalista. Esta es una hipótesis, no una definición. Tan es así que es verdadera tan sólo en primera aproximación, ya que hay factores psicológicos y políticos, no sólo económicos, que determinan los niveles de bolsa. Una definición, en cambio, es verdadera 
por estipulación o convención. Ejemplo: Oligopolio es una economía o un mercado controlado por unas pocas empresas.

\section{Estructura y estructuralismo}

La palabra "estructura" está de moda pero no siempre designa un concepto claro. Por ejemplo, cuando se habla de la "visión estructural de los problemas sociales", o de "historia estructural", habitualmente no se entiende de qué se trata. En sentido estricto (matemático) una estructura es un conjunto arbitrario de elementos dotado de una o más relaciones, operaciones $o$ funciones. Por ejemplo, si $P$ es el conjunto de paises y $R$ una relación de dependencia (en algún respecto), entonces el par ordenado $\langle P, R\rangle$ es una estructura representativa de la dependencia en el respecto en cuestión. Correspondientemente, una "visión estructural" de la dependencia, o de cualquier otro hecho, sería una conceptualización del hecho en términos de, o con ayuda de, estructuras matemáticas precisas, o sea, un modelo matemático del hecho. Los únicos estructuralistas en sentido estricto serían entonces los matemáticos puros y aplicados, entre ello los sociólogos matemáticos. Sin embargo, no parece ser éste el sentido de la frase "visión estructural" en los escritos de los sociólogos dominados por la escuela estructuralista. Parecería que se trata de una doctrina más bien que de un método, y que la doctrina se reduce a la tesis dudosa de que lo permanente y universal, antes que lo transitorio y regional, debiera estar en el foco de las ciencias del hombre. Pero, dado el estilo oscuro de los escritos en cuestión, es difícil aseverar si esto es lo que se proponen decir.

\section{Explotación}

La noción de explotación, de origen ideológico, se ha incorporado a la sociología de inspiración marxista así como a los estudios sobre dependencia económica de los pafses periféricos respecto de las metrópolis. Es una noción cualitativa y poco clara que puede cuantificarse y con ello aclararse sin dificultad en términos de los conceptos de costo y beneficio, que son clásicos en la economia.

El costo puede concebirse como una función que asigna un número positivo a cada operación de producción. En otras palabras, $C: O \rightarrow R^{*}$, donde " $C(z)=r$ ", para $z$ en el conjunto $O$ de operaciones y $r$ en el conjunto $R *$ de números reales, se interpreta como el costo de $z$.

Por su parte, el beneficio que obtiene la persona (fisica o jurídica) $x$ de la persona (f́́sica o jurídica) y que realiza o permite realizar la operación $z$, puede concebirse como el valor de la función $B: P \times P \times O \rightarrow R^{*}$ que asigna un número positivo $w$ a la terna ordenada $\langle x, y, z\rangle$ perteneciente al con- 
junto de ternas $P \times P \times O$. Ya tenemos lo necesario para introducir la noción de grado de explotación.

Consideremos primero la diferencia $B(x, y, z)-C(z)$ entre el beneficio que obtiene $x$ de $y$ por la operación $z$, y el costo que ocasiona $z$ a $x$. Esta diferencia es el beneficio neto, para $x$, de la operación $z$ ejecutada o permitida por $y$. Compárese ahora este beneficio neto con el beneficio $B(y, x, z)$ que obtiene $y$ como retribución por ejecutar o permitir la operación $z$. Este beneficio total puede ser igual a la suma de un salario más beneficios sociales (salud pública, educación, pensión de la vejez, etc.) o puede ser una mera regalía, como ocurre cuando una empresa paga derechos por la explotación de un recurso natural. Es decir, fórmese la diferencia

$$
E(x, y, z)=B(x, y, z)-C(z)-B(y, x, z) .
$$

Si esta diferencia es nula, se trata de un trato justo por el cual se benefician mutuamente $x$ e $y$. Si la diferencia es negativa, el que carga con el costo, o sea $x$, sale perjudicado. Y si la diferencia es positiva, quien sale perdiendo es $y$. En este último caso, o sea, cuando la diferencia es positiva, puede llamársela el grado de explotación de que es objeto $y$ por parte de $x$ en el respecto $z$.

Si el que ejecuta o permite la operación $z$ es todo un grupo social $g$ (comunidad o nación), debemos sumar los beneficios de uno y otro lado, resultando así

$$
E(x, g, z)=\underset{y_{E} J}{\Sigma} B(x, y, z)-C(z)-\sum_{y_{E} g} B(y, x, z) .
$$

Para computar el grado de explotación, sea individual, sea colectiva, es preciso a) estimar los costos y beneficios en un periodo relativamente largo, para evitar que pesen excesivamente los costos de instalación, y $b$ ) incluir en los beneficios que recibe y no solamente los directos (p. ej. en forma de salarios) sino también los indirectos o sociales.

Presumiblemente, el empleo de la noción de grado de explotación que acabamos de introducir -o de otra acaso más adecuada pero igualmente precisa- contribuiría a una mejor descripción de las situaciones de hecho así como a la formulación de modelos matemáticos de sistemas de explotación.

\section{Grupo social}

El concepto de grupo social (en particular de clase social) es tan básico que pocos sociblogos se ocupan de aclararlo. Un análisis posible y elemental es el que sigue. Sea $C=\langle H, R\rangle$ una comunidad que consta de un conjunto $H$ de personas relacionadas por relaciones sociales comprendidas en el 
conjunto $R$. Algunas de estas relaciones son relaciones de equivalencia (reflexivas, simétricas y transitivas). Ejemplos: ocupación similar, nivel cultural parecido, pertenencia a la misma iglesia, y poder económico similar. Cualquiera de estas relaciones de equivalencia social induce una partición del conjunto $H$ en subconjuntos homogéneos y mutuamente disjuntos: cada uno de éstos es un grupo social. Por ejemplo, si dos miembros de $H$ están ligados por la relación $R_{i}$ perteneciente a $R$, entonces son equivalentes en el respecto $\boldsymbol{R}_{\boldsymbol{k}}$, o sea, pertenecen ambos al mismo grupo social de la partición inducida por $R_{i}$ (aun cuando no pertenezcan a los mismos grupos sociales producidos por otras relaciones pertenecientes a $R$ ). Habrá tantas particiones, o sea, tantas colecciones de grupos sociales, como relaciones de equivalencia social. La totalidad de estas particiones puede llamarse la estructura social de $H$.

Lo que vale para los grupos sociales en general vale para las clases sociales. En este caso la relación de equivalencia es la de igual poder económico. El concepto de poder económico puede definirse a su vez en función del número de productores controlados. El poder económico $P(x, y)$ que ejerce la familia $x$ en la comunidad $y$ es igual al número de miembros de la comunidad $y$ que trabajan para $x$ (o cuyas actividades económicas controla o influye $x$ ) dividido por el número total de familias de $y$ menos uno. (O sea, primero se hace la partición de la comunidad en familias, luego se le asigna a cada una un poder económico.) Las familias que ejercen un poder económico similar (o comprendido entre dos cotas dadas) pertenecen a la misma clase social. En otras palabras, si $a$ y $b$ son dos números reales no nulos, entonces el conjunto de familias para las cuales hay al menos un $x$ tal que el máximo de $P(x, y)$ está comprendido entre $a$ y $b$, es una clase económica. Ejemplo: sea una comunidad que consta de 13 familias agrupadas de la manera siguiente:

1 familia dominante, que ejerce el poder económico 12/12;

2 familias de clase media, una de las cuales controla económicamente a 3 familias y la otra a 7 , pero que son mutuamente independientes, de suerte que el poder económico en esta capa social está comprendido entre $3 / 12$ y $7 / 12$;

10 familias de clase ínfima, que ejercen poder económico nulo.

Se objetará que esta manera de concebir las clases sociales es simplista. Concedido. Pero es preferible un concepto simple y claro a una palabra que no designa ningún concepto preciso, y que sin embargo se emplea una y otra vez. Una vez que se ha alcanzado una claridad inicial se puede aspirar a una mayor complejidad, en tanto que la insistencia en que la realidad es demasida compleja para ser apresada en fórmulas matemáticas no es sino una forma de oscurantismo. De oscurantismo y a veces también de defensa de la propia ignorancia. 


\section{Ideologia}

Las ideologías son de dos tipos: religiosas y sociopolíticas. Una ideología sociopolítica es una visión del mundo social: es un conjunto de creencias referentes a la sociedad, al lugar del individuo en ésta, al ordenamiento de la comunidad y al control político de ésta. Estas creencias pueden agruparse en cuatro clases:

a) afirmaciones ontológicas acerca de la naturaleza de la persona y de la sociedad: qué clase de entes son las personas (materiales, espirituales, o mixtos), de qué modo se combinan para formar comunidades, y qué son éstas (animales, culturales, o mixtas);

b) afirmaciones acerca de los problemas económicos, culturales y políticos de las comunidades de diversos tipos: en qué consisten dichos problemas y cuáles son sus prioridades;

c) juicios de valor acerca de las personas y de sus actos sociales, así como de las organizaciones y sus metas: qué es bueno y qué es malo para la sociedad;

d) un programa de acción (o de inacción) para la solución (o la conservación) de los problemas sociales y la obtención de un conjunto de metas individuales y sociales.

La consideración de un ejemplo ayudará a comprender cómo funciona la ideología y cómo se relaciona con otros campos. Si se pregunta: ¿De qué viven y cómo viven los campesinos sin tierra del estado $X$ ?, se pide una investigación científica o los resultados de la misma. En cambio, si se pregunta: ¿Es justo y conviene a la sociedad en su conjunto que haya campesinos sin tierra en una zona donde algunas familias acaparan grandes extensiones de tierra?, esta pregunta clama por una respuesta ideológica. Finalmente, si se pregunta: ¿Qué debiera hacerse para que los campesinos desposeidos adquieran tierra?, se plantea un problema político fundado sobre una respuesta negativa a la pregunta anterior. Pero la respuesta a este problema político debiera a su vez fundarse sobre un estudio científico de la cuestión de la tierra: es preciso saber cuánta tierra hay, cuántos campesinos capaces de cultivarla, qué necesitarían para cultivarla efectivamente mejorando su suerte individual y asegurando el abastecimiento de la población urbana, etc. En otras palabras, una solución adecuada al problema político requiere una investigación en sociologia aplicada o de programa. En suma, la cuestión agraria tiene varias facetas y exige que se le trate desde otros tantos puntos de vista: sociología básica o pura, ideología, sociologfa aplicada, y política. En rigor debiéramos incluir también a la filosofía, no porque esté en condiciones de dar respuestas precisas a los problemas sino porque toda investigación, sea básica, sea aplicada, es guiada por supuestos filosóficos. Las relaciones mutuas entre las cinco áreas mencionadas se exhiben en la figura siguiente: 


\begin{tabular}{|l|ll|}
\hline $\begin{array}{l}\text { Política } \\
\uparrow\end{array}$ & Ideología \\
\hline $\begin{array}{l}\text { Sociología } \\
\text { aplicada } \\
\uparrow\end{array}$ & & \\
\hline $\begin{array}{l}\text { Sociología } \\
\text { básica }\end{array}$ & $\rightarrow$ & Filosofía \\
\hline
\end{tabular}

Nótese la diferencia entre una ideología y una teoría sociopolftica: una teoría está constituída por hipótesis, no por afirmaciones dogmáticas, y no contiene juicios de valor ni programas de acción. (En cambio los juicios de valor y los programas de acción figuran en sociología aplicada.) Además, por lo común una ideologfa no es producto de la investigación básica ni cambia con los resultados de ésta: hasta ahora las ideologfas han sido bastante resistentes a las novedades científicas. Una ideologia puede cambiar, pero tan sólo en detalles: si un ismo cambiara radicalmente dejaría de ser ese ismo. Además, los cambios que se producen en una ideologia son comúnmente introducidos por algún líder carismático en lugar de ser resultado de investigaciones realizadas por la comunidad de investigadores. La ideología, en suma, no tolera la crítica, no practica la autocrítica, y no está al día con los avances de la ciencia social. Antes al contrario, los movimientos que sustentan una ideología practican la crítica, sea externa (a los demás), sea interna (a sí mismos) sobre la base de una ideologia rigida. Se apoya o condena a X por ser $\mathrm{X}$ fiel al ismo en cuestión.

Las ideologías descritas en el párrafo anterior son las ideologias no científicas. Pero una ideologia no es necesariamente ajena a la ciencia. En principio es concebible una ideología adecuada a las ciencias y a la realidad social de una determinada área. Por ejemplo, una ideologia que preconice medios viables para incrementar la participación popular en la producción económica y cultural, aś́ como en la conducción política de una comunidad - sea, que propicie la democracia integral - puede considerarse cientifica porque la sociologia nos enseña que la participación múltiple (no sólo politica) y constante (no esporádica) es la única garantía de cohesión social y, por lo tanto, de estabilidad tanto como de evolución. En cambio, las.ideologías que preconizan la dictadura indefinida de un grupo social sobre otros no son solamente antipopulares sino también anticientificas, porque la gente sometida termina, sea por rebelarse, sea por degradarse y por arrastrar en su degradación a los opresores. En suma, en principio es posible diseñar ideologias cientificas, sistemas de creencias fundadas en el estudio científico de la realidad social, asf como en un sistema de valores auténticamente democrático. 
De hecho no hay aún ideologías plenamente cientificas: las que se disputan el mundo son más o menos conservadoras (al par que la ciencia es revolucionaria), más o menos utópicas (en tanto que la ciencia es realista), más o menos incapaces de aprender de la experiencia (al par que las ideas científicas no cesan de ser corregidas a la luz de la experiencia y de la crítica), y rara vez fundan sus programas de acción sobre un estudio científico de la realidad social. Por estos motivos, aunque hay que propiciar la formación de ideologías científicas - actitud más realista y constructiva que predicar la pureza de la sociología - es preciso admitir que estamos aún lejos de tales ideologías ilustradas.

Igualmente, es preciso reconocer que la ciencia social jamás está libre de ideologia: que, si bien un dato o una teoria son verdaderos o falsos independientemente de consideraciones ideológicas, el planteo de problemas científicos, el diseño de planes de investigación, y la evaluación de resultados, tienen lugar en un marco conceptual que incluye elementos ideológicos. Por ejemplo, quien reconozca la existencia e importancia de la desigualdad social tratará - si tiene una mentalidad cientifica - de precisar los conceptos de desigualdad (económica, cultural y política), de hallar indicadores fidedignos de desigualdad, y de buscar datos para dar valores a tales indicadores. En cambio, quien sostenga que no hay inequidad social en una comunidad dada, o que si la hay no importa, no emprenderá tal investigación. En resumen, aunque la ideología no es creadora sino más bien consumidora, puede inspirar la investigación científica u obstaculizarla. De aquí que no conviene a los intereses de la ciencia seguir insistiendo en una imposible neutralidad ideológica. La neutralidad ideológica no es sino hoja de parra política. Lo que cabe no es cerrar los ojos a la realidad de la interdependencia entre ciencia e ideologia, sino bregar por someter a la ideologia al control de la ciencia, asi como por utilizar la ciencia para resolver los problemas prácticos.

\section{ro. Infraestructura y superestructura}

Es común la distinción entre infraestructura material y superestructura ideal o cultural. Esta dicotomía es traducción a la esfera social del viejo dualismo religioso y filosófico entre el cuerpo y el alma. Este dualismo es compartido por materialistas históricos y por idealistas. La diferencia entre ellos es que los primeros sostienen que la infraestructura genera y domina a la superestructura, en tanto que los idealistas invierten la relación de dependencia.

En realidad no existe tal separación entre cuerpo y alma, entre infraestructura material y superestructura ideal o cultural. Lo que hay en realidad son personas que sienten y piensan así como digieren y caminan. Lo que hay en realidad son personas que producen y consumen bienes materiales, 
tales como tortillas de maíz, y bienes culturales, tales como conversaciones. Toda actividad económica tiene componentes culturales; incluso la economía doméstica requiere el aprendizaje, la transmisión y la aplicación de recetas. Toda actividad cultural tiene componentes económicos; incluso el garabato de un niño requiere papel y lápiz, que son mercancfas. $Y$ toda actividad política está comprendida entre la esfera económica y cultural.

El auténtico materialismo no afirma que lo material determina a 10 ideal, sino que niega la existencia de lo ideal como cosa y lo afirma como actividad o función de las personas. Lo que suele llamarse "ideal" es una abstracción: se trata de una actividad de seres de carne y hueso que se valen de herramientas materiales tales como hachas o computadoras.

La división entre infraestructura y superestructura no tiene ni siquiera utilidad heurística. Mucho más sugerente es la distinción de tres subsistemas dentro de toda comunidad, por primitiva que sea: la economía, la cultura y la política. Cada uno de estos subsistemas, lejos de ser un conjunto de actividades desencarnadas, es un conjunto de personas que ejercen ciertas actividades. Más aún, lejos de ser ajenos entre sí, estos subsistemas se intersectan. Por ejemplo, un técnico agrícola pertenece tanto a la economía como a la cultura; un inspector de escuelas pertenece simultáneamente a la cultura y a la política; y un empleado de hacienda pertenece tanto a la política como a la economía. Este análisis de un sistema social en tres subsistemas es más realista y más sugerente que el dualismo infraestructura-superestructura.

\section{Ley y causa}

Algunos pensadores, desde Heráclito hasta ciertos economistas contemporáneos, conciben las leyes naturales o sociales como causas: hablan p. ej. de los efectos de una cierta ley de formación de precios, como quien hablase de los efectos de beberse una botella de vino. Esta manera de hablar se justifica en el contexto de la filosofía heraclítea, en la que la razón o logos se identifica con la ley y la norma. No es ésta la concepción contemporánea de ley natural o social (a diferencia de la ley positiva o norma jurídica). Según esta concepción, las leyes no existen separadamente de las cosas sino que son el moddo constante (pauta) de ser y devenir de las cosas mismas. Por lo tanto, Ias leyes no son causas, luego no tienen efectos. A lo sumo puede decirse que, en primera aproximación, hay leyes causales que relacionan causas con efectos. Lo que tiene consecuencias (lógicas), aunque no efectos (materiales), son los enunciados legales, o sea, las fórmulas mediante las cuales expresamos (en forma aproximada) dichas pautas objetivas. 


\section{Lógica de los hechos}

Los autores influidos por Hegel suelen hablar de la lógica de los acontecimientos y procesos. Se trata de una expresión desgraciada, que posee sentido solamente en un sistema idealista ( p. ej. el de Hegel) en el cual los hechos no son sino corporizaciones del espiritu (sea subjetivo, sea objetivo o absoluto). En rigor no hay lógica de los hechos así como tampoco hay física de los conceptos. Lo que hay son mecanismos de los hechos (p. ej. del desarrollo económico), y tales mecanismos son parte de las tendencias y de las leyes. Nada de esto tiene que ver con la lógica en sentido estricto, que es la teoría de la estructura del argumento deductivo. No hay una lógica social como tampoco hay una lógica química: existe solamente la lógica a secas (o simbólica, o matemática). No es ésta una mera disputa verbal sino que lo es metodológica. En efecto, si existiese una lógica de los hechos, puesto que la lógica es a priori (previa a la experiencia), entonces el estudio de los hechos podría hacerse independientemente de nuestra experiencia con éstos. Que es, precisamente, el motivo por el cual Hegel hablaba de la "lógica objetiva".

\section{Matemática y ciencias sociales}

La sociología clásica conocía un solo capítulo de la matemática: la estadística matemática. Aun hoy son numerosos los sociólogos que creen que su misión consiste en coleccionar datos, con prescindencia de toda teoría, y en llamar en su ayuda a un estadígrafo que elabore tales datos y "extraiga conclusiones". Hoy día el sociólogo debe recurrir no sólo a la estadística matemática para elaborar los datos y controlar las hipótesis: debe recurrir también a las demás ramas de la matemática para elaborar sus ideas, del mismo modo en que vienen haciéndolo las ciencias naturales desde hace tres siglos y medio.

Las funciones de la matemática en las ciencias sociales son por lo menos las siguientes:

a) la matemática provee a todas las ciencias un esqueleto formal prefabricado que puede rellenarse con cualquier contenido empírico compatible con la estructura formal;

b) la matematización de los conceptos y de las proposiciones incrementa la exactitud y por lo tanto la claridad de las ideas;

c) una teoría matemática posee un poder deductivo ajeno a una doctrina verbal: en ésta las inferencias son laboriosas y a menudo inseguras, ya que no se sabe bien cuáles son las premisas;

d) la precisión y el poder deductivo aumentan la verificabilidad de la teoría: se facilita la derivación de conclusiones exactas, las que se pueden confrontar con los datos empíricos; 

tizar;

e) la teoría se puede ordenar mejor y, en particular, se puede axioma-

f) el mejor ordenamiento lógico y la facilitación de la contrastación empírica hacen a su vez más fácil la comparación de la teoría dada con teorías rivales;

g) se resuelven automáticamente, y sin recurso a ideología alguna, viejas controversias filosóficas que han obstaculizado la marcha de la ciencia, tal la disputa entre el individualismo y el colectivismo metodológico en las ciencias sociales. Este último punto merece un párrafo aparte.

Los individualistas sostienen que todo discurso sociológico debiera referirse en última instancia a los individuos que componen una sociedad, en tanto que los colectivistas (o totalistas) afirman que hay propiedades sociales colectivas inexplicables en términos de propiedades individuales. La sociología matemática no se plantea esta disyuntiva si emplea las herramientas conceptuales adecuadas. En efecto, una sociedad puede representarse por una estructura relacional $S=\langle H, R\rangle$, donde $H$ es un conjunto de individuos y $\boldsymbol{R}$ un conjunto de relaciones (sociales) entre miembros de $H$. Una sociedad no es, pues, un mero conjunto (o "suma") de individuos, ni es tampoco un ente supraindividual que planea por encima de éstos, ya que las relaciones que ligan entre sí a los miembros de una sociedad no existirían sin éstos, ni éstos serían tales miembros si no estuvieran relacionados socialmente entre sí. Una sociedad no es ni más ni menos que un conjunto de individuos que viven en sociedad, o sea, que están conectados por relaciones sociales. Como se ve, para alcanzar esta síntesis de los dos polos tradicionales no se utilizó ninguna herramienta matemática complicada: bastó la noción de estructura relacional, o conjunto estructurado por un conjunto de relaciones. Se trata de una noción simple y cualitativa pero precisa, cuya aplicación muestra el absurdo del individualismo y del colectivismo metodológicos.

\section{Método dialéctico}

Entre los sociólogos del Tercer Mundo está de moda hablar del método dialéctico, pero nadie parece saber en qué consiste: cuáles son sus reglas, a qué y cómo se les aplica, ni cómo se controla su aplicación. Existe ciertamente una ontología o metafísica dialéctica, o más bien dos, la una idealista y la otra materialista (en la medida en que el materialismo es compatible con la dialéctica, lo que es dudoso). Pero se trata de un conjunto de hipótesis muy generales, llamadas "leyes de la dialéctica", acerca del devenir, en particular del desarrollo sociohistórico. Estas hipótesis más o menos precisas no constituyen un método, esto es, un procedimiento para hacer o estudiar algo. Constituyen una doctrina.

No hay pues método dialéctico sino más bien un enfoque dialéctico de 
problemas, sean teóricos, sean prácticos, consistentes en presuponer la ontología dialéctica e intentar encajar en ella los objetos de interés. Tal enfoque se caracteriza por la búsqueda de polaridades $(q . v$.) y por la exageración de la importancia de los conflictos (en la naturaleza, en la sociedad o en el pensamiento), a expensas de la cooperación y de cualesquiera otros mecanismos de cambio. Como se indica en el artículo Polaridad, este enfoque es típico de una etapa primitiva del pensamiento. La ciencia no se limita a buscar polaridades sino que se esfuerza por encontrar pautas objetivas (leyes), las que rara vez son polares. Y, aun en el caso en que el conflicto es real, es de una complejidad tal que desborda el marco polar.

Tómese, por ejemplo, la teoría de Volterra de la lucha interespecífica, aplicable tanto a poblaciones animales como a comunidades humanas. Supongamos que el sistema de interés esté constituido por dos poblaciones, una de las cuales vive a costillas de la otra. El ejemplo clásico es el de una población de liebres depredada por una población de zorros; pero la teoria vale igualmente para el par terratenientes-campesinos. Llamemos $H$ a la población huésped y $P$ a la población parásita. La evolución de estas poblaciones en el curso del tiempo está descripta por el par de ecuaciones diferenciales

$$
\frac{d H}{d t}=\left(a_{11}+a_{12} P\right) H \quad \frac{d P}{d t}=\left(a_{21}+a_{22} H\right) P
$$

donde los coeficientes $a_{11}$ y $a_{22}$ son números reales positivos, y los demás reales negativos. Esta teoria describe el conflicto en detalle y, a diferencia de una "interpretación dialéctica", prevé las conocidas oscilaciones de población. Ciertamente, la teoría no es sino parcialmente verdadera. Pero es susceptible de corrección y, en todo caso, provee una primera aproximación. Por este motivo es parte integrante de la ecologia de las poblaciones, en tanto que la doctrina dialéctica de los conflictos es imprecisa e incapaz de formular predicciones comprobables.

Para terminar: la frecuencia con que aparecen los términos "proceso dialéctico" y "método dialéctico" en un campo de estudios es un buen indicador del bajo grado de desarrollo de éste. Los físicos, químicos, biólogos, psicólogos y sociólogos matemáticos no hablan de objetos dialécticos ni dicen em. plear el método dialéctico: emplean el método científico, que les permite formular concepciones precisas y comprobables. Si lo que se proponen los pensadores dialécticos es tan sólo subrayar el carácter cambiante de todas las cosas y la naturaleza conflictiva de algunos procesos, no necesitan salirse de la ciencia, ya que ésta estudia las leyes del cambio y, en particular, de la competencia. La dialéctica es dinamicista pero no científica; la ciencia no es dialéctica pero es dinamicista y, además, clara y a veces verdadera. 


\section{Polaridad}

Los historiadores de las ideas han mostrado que es característica del pensamiento arcaico y aun antiguo el reducirlo todo a pares de opuesto: día-noche, mortal-inmortal, comestible-incomible, móvil-inmóvil, etc. Esta característica se conserva en la primera fase del tratamiento científico de un problema: así como el neurofisiólogo de principios de siglo intentaba reducirlo todo a un juego de excitaciones e inhibiciones, el sociólogo del subdesarrollo cae en la tentación de explicarlo todo en términos de dependencia e independencia y de pares opuestos similares.

Sin duda algunos sistemas reales, sean físicos, sean culturales, presentan características polares. Pero también presentan otras que no lo son. La ciencia moderna ha mostrado que la realidad no cabe dentro de los esquemas polares, sea de Pitágoras, sea de Hegel: los sistemas polares son la excepción, no la regla. Aun así, la polaridad, cuando existe, no abarca todos los aspectos de un sistema. El estudio de un interruptor eléctrico comienza, no termina, con la observación de que puede estar abierto o cerrado. Y la mecánica no se limita a clasificar los cuerpos en móviles o inmóviles.

A medida que crece el número de sociólogos científicos en los países socialistas, crece el número de los sociólogos filosóficos ("humanistas") en los países capitalistas y del tercer mundo. Entre ellos hacen más ruido que luz los que sostienen inspirarse en la filosofía dialéctica. Esta filosofía comienza por distinguir los componentes o aspectos mutuamente contradictorios del sistema en cuestión, y encuentra en esta oposición el motor de su desarrollo (cuando no de su estancamiento). Este tipo de explicación es tan primitivo como la dicotomización que la precede e igualmente incompatible con una visión científica del mundo. Imagínese un físico que intentase explicar el funcionamiento de un motor de automóvil en términos de las oposiciones calor-frío, contracción-dilatación y movimiento-reposo en lugar de valerse de la termodinámica y de la mecánica.

Ciertamente, cuando hay polaridad u oposición también hay cambio o al menos equilibrio inestable. Pero a veces no hay tal oposición y sin embargo hay desarrollo, como ocurre con los procesos físicos, biológicos y sociales de cooperación. Todo cambia, ciertamente, pero no todo cambio se debe a una "lucha de contrarios". Y cuando hay tal lucha no se la describe adecuadamente en términos simplistas (polares) sino empleando conceptos mucho más ricos, tal como lo hacen la teoría matemática de la competencia interespecifica y la teoría de los juegos.

Entre los sociólogos, ideólogos y filósofos del Tercer Mundo está de moda emplear la expresión " $x$ es dialéctico", pero ninguno de ellos la explica. Parecería que, al igual que los filósofos intuicionistas, dichos pensadores son incapaces de hacer un esfuerzo de análisis conceptual. Lo menos que puede 
hacerse es intentar una definición tal como ésta: Un objeto $x$ es dialéctico si y sólo si existen al menos otros dos objetos, $u$ y $v$, tales que a) $u$ y $v$ forman parte de $x, \mathrm{y}$ b) $u$ y $v$ se oponen entre sí en algún respecto. Con todo, esta es una seudoexactitud, ya que no está claro qué significa "oposición": ¿incompatibilidad, fuerzas encontradas, o qué? Mientras no se aclare ese término oscuro, casi siempre metafórico, convendrá abstenerse de emplearlo. No es posible hacer girar toda una filosofía en torno a la oscuridad. Ni es posible obstinarse en reducir la complejidad real a polaridades y luchas de contrarios, como lo hacían los presocráticos. Estamos a fines del siglo $\mathrm{xx}$, no a fines del siglo $\mathrm{v}$ antes de nuestra era.

Véase Método dialéctico.

\section{Sentido y significación de los hechos}

Max Weber hablaba del sentido de los actos humanos, y muchos sociólogos influidos por él hablan de la significación de los hechos sociales. Esta terminología es equívoca y evitable. En los escritos de Weber la palabra "sentido" puede reemplazarse por "finalidad". Por ejemplo, en lugar de decir "el sentido de la lucha por la independencia" puede decirse "la finalidad de la lucha por la independencia".

No está claro en cambio qué puede significar la expresión "la significación del hecho $x$ ", a menos que se quiera decir "la contribución del hecho $x$ al hecho posterior $y "$, donde $y$ es el hecho principal que se estudia o bien una finalidad a lograr. No conviene violar la etimología de "significación", que se refiere a signos, no a hechos extralingüísticos: se puede hablar legitimamente de la significación de un signo o símbolo escrito o hablado, pero no de la significación de los acontecimientos (a menos que se pretenda que ciertos hechos son signos o augurios de otros). Dicho en otros términos: es menester no confundir el estudio sociológico de las actividades humanas con la semiótica.

\section{Sociologia}

Una de las caracteristicas típicas del subdesarrollo es que su propia sociología es subdesarrollada. Es decir, los trabajos que se ocupan del subdesarrollo alcanzan rara vez un nivel científico moderno. En general, en los países del Tercer Mundo somos muy generosos en el empleo de la expresión "ciencia social". Por ejemplo, solemos llamar Facultad de Ciencias Sociales a lo que casi siempre es una Escuela de Ideologías Sociales. Y la mayor parte de nuestras revistas de Ciencias Sociales debieran llamarse Revistas Sobre Cuestiones Sociales. En efecto, el tema no lo es todo: un mismo tema puede 
abordarse, sea científicamente, sea de otro modo. En particular, la literatura sobre temas sociales puede clasificarse del modo siguiente: sonal.

a) Impresionismo: apreciaciones fundadas tan sólo en la experiencia per-

b) Critica social: fundada en datos parciales o en consignas ideológicas.

c) Ideologia: juicios de valor y programas de acción.

d) Sociologia literaria o filosófica: descripciones y análisis impresionistas fundadas en datos escasos y que no llegan a ser teorías.

e) Sociología cientifica: conjuntos de hipótesis veríficables y de datos fidedignos. La sociología científica ha pasado por tres etapas de desarrollo, todas las cuales coexisten hoy día:

i) sociografía, o descripciones precisas pero superficiales por la falta de teorias propiamente dichas;

ii) sociología clásica: datos no muy refinados y teorías verbales (no matemáticas);

iii) sociologia avanzada: investigación empírica combinada con modelos matemáticos y un comienzo de experimentación.

Lo que hemos llamado sociografía es particularmente patente en la antropología social (o cultural) desde Boas hasta nuestros días. Los sociografos son los juntadatos cuidadosos que desconfían de las teorias y que, por lo tanto, a menudo coleccionan datos poco interesantes, poco reveladores de los mecanismos íntimos del cambio social, mecanismos que siempre están ocultos a la observación directa y que sólo pueden conjeturarse. La sociologia clásica comprende las obras de Marx, Durkheim, Weber, Veblen, Leslie White y Myrdal, para citar sólo a los más eminentes y mejor conocidos. Es la época de las grandes síntesis teóricas fundadas sobre datos fehacientes. Pero las teorías de ese periodo son meramente verbales y por ende imprecisas, y los datos son los que buenamente quieran proporcionar las oficinas estadísticas: no hay modelos matemáticos y por lo tanto las ideas son un tanto imprecisas y la interpertación de los escritos es discutible. Finalmente, la sociologia de avanzada se caracteriza por proponer modelos matemáticos, los que sugieren la búsqueda de datos de tipo insospechado, así como por el diseño de uno que otro experimento social en gran escala. Descuellan en esta etapa, entre otros, Pareto, Lazarsfeld, Coleman, Dahl, Harrison White, y Boudon, por no mencionar a los economistas matemáticos, que les preceden en casi un siglo.

La sociología de avanzada está confinada a unos pocos países: en los demás se cultivan aún la sociografía y la sociologia clásica. Más aún, en los países de la periferia científica suele haber resistencia abierta a la sociología contemporánea, y ello por motivos filosóficos. Los positivistas, que sostienen apegarse a los datos (superficiales), rechazan toda teoría y se quedan por lo tanto en la sociografía. Y los sociólogos de formación humanista se refugian en la escuela idealista, según la cual las ciencias sociales son ciencias del es- 
piritu (Geisteswissenschaften) y, por lo tanto, ajenas al método científico, el que sería aplicable tan sólo a la naturaleza. (Además, la verdad es que casi todos ellos han sido entrenados para leer y comentar textos más que para investigar la realidad social y teorizar con ayuda de la matemática.) Esta resistencia se parece a la que experimentaban los escolásticos ante los trabajos revolucionarios de Galileo, al decirle: "No tenemos necesidad de tu telescopio: nos bastan los ojos que Dios nos ha dado. Ni tenemos necesidad de tus fórmulas matemáticas: nos bastan los textos escritos en lenguaje ordinario, que nos han legado Aristóteles y sus comentaristas."

\section{Sociologia latinoamericana}

Casi toda la producción sociológica, psicosociológica y politológica referente a Latinoamérica exhibe, en mayor o menor grado, diversos defectos conceptuales. Toda ella usa ( $y$ a veces abusa de) nociones oscuras o imprecisas. Por consiguiente las hipótesis que las contienen son ellas mismas oscuras e imprecisas, luego difícilmente comprobables. Por añadidura esas hipótesis no se presentan agrupadas en teorias propiamente dichas sino, más bien, en doctrinas o "interpretaciones".

Como consecuencia de la debilidad metodológica apuntada, las "interpretaciones" del desarrollo y de la dependencia no proveen descripciones exactas de la situación de dependencia ni del proceso de desarrollo. A fortiori no dan una explicación adecuada ni permiten formular predicciones precisas que las pongan a prueba o que sirvan para la acción política. En resumen, la producción en cuestión, aunque interesante y a menudo rica en sugerencias, dista de ser científica en la plena acepción de la palabra. En el mejor de los casos dichos trabajos son un punto de partida para una investigación riguro. sa, y en el peor son trabajos periodisticos o aun ideológicos.

Los defectos anotados no son exclusivos de los estudios latinoamericanos y no tienen nada que ver con la carencia de datos empíricos. Son defectos conceptuales característicos del pensamiento sociológico tradicional anterior al nacimiento de la sociologia matemática. Son los mismos males conceptuales que aquejaban a la física anterior a Galileo. Estos defectos se corrigen con una dosis de análisis conceptual y metodológico y otra de matemática elemental. Se trata, en suma, de dilucidar ciertos conceptos y de exactificar otros, así como de construir teorías propiamente dichas y de hacerlo con ayuda de la herramienta conceptual universal, a saber, la matemática.

Semejante reorientación conceptual en la investigación de la sociedad latinoamericana llevaría a su vèz a una reorientación de la investigación empírica. Se buscarían datos relevantes a teorías bien formuladas, en lugar de acumular datos porque sí o para alimentar hipótesis imprecisas. Y si se dispusiera de teorías propiamente dichas, y más aun de teorías confirmadas, 
acerca de la dependencia y del subdesarrollo, se las podría aplicar: se podría discutir más eficazmente las medidas prácticas que debieran tomarse para lograr la independencia y orientar el desarrollo en beneficio de la propia América Latina. Mientras tanto se seguirá improvisando o inspirándose en ideologías. De aquí la enorme importancia práctica de la filosofía (avanzada) de la ciencia (avanzada).

\section{Teoria, marco teórico, doctrina, interpretación}

En las ciencias sociales hay tendencia a dignificar con el nombre de teoria a cualquier montón de opiniones, por desconectadas que estén y por infundadas que sean. Casi siempre se trata de meros marcos teóricos o de doctrinas. Vemos las diferencias.

La noción de marco teórico o conceptual, o simplemente contexto, puede caracterizarse como sigue. Un marco teórico (o contexto) es un conjunto de proposiciones referentes a un mismo dominio (p. ej. sociedades humanas) y tales que contienen ciertos conceptos (p. ej. los de clase social y anomie) que constituyen un grupo homogéneo, en el sentido de que todos ellos se refieren al mismo dominio. Más aún, en virtud de esta referencia común, un contexto, sin ser una teoría propiamente dicha, posee un grado de organización muy superior al de una colección de proposiciones tomadas al azar. En efecto, las proposiciones de un marco teórico o contexto se pueden negar y combinar, sea disyuntiva, sea conjuntivamente, sin que resulten proposiciones ajenas al contexto. En otras palabras, las proposiciones de un contexto dado constituyen un conjunto cerrado respecto de las operaciones lógicas. Un contexto sirve entonces de materia prima para la elaboración de teorías, ya que cada una de éstas se obtendrá seleccionando proposiciones del contexto y, en particular, guardando solamente aquellas que constituyan un conjunto coherente (no contradictorio).

Una posible definición formal de contexto o marco teórico es ésta: " $\mathrm{La}$ terna ordenada $C=\langle S, P, R\rangle$ es un contexto o marco teórico si y solamente si $S$ es un conjunto de proposiciones tales que a) $S$ es cerrado respecto de las operaciones lógicas, b) $S$ contiene solamente predicados comprendidos en $P$, y c) todos los predicados comprendidos en $P$ se refieren a $R$." (En rigor esta definición vale solamente para predicados monádicos o unarios, tales como el atributo de ser subdesarrollado. Se la generaliza suponiendo que $R$ es una familia de conjuntos de objetos, y que cada miembro de $P$ se refiere a uno o más miembros de esta familia. Por ejemplo, un contexto para el estudio de la dependencia contendrá, entre otros, los conceptos de país central y de país periférico, así como el concepto de dependencia de un país respecto de otro. El concepto de dependencia es una relación binaria entre miembros de ambos conjuntos.) 
Según el diccionario, una doctrina es un cuerpo de ideas suceptible de ser transmitido o enseñado. En cambio, una teoria es una doctrina muy especial: es un sistema hipotético-deductivo, o sea, un cuerpo de ideas organizado lógicamente. Más precisamente, una teoría es un conjunto de proposiciones, todas ellas referentes a un asunto dado (p. ej. el subdesarrollo), y tales que cada una de ellas es, o bien una premisa (p. ej. una hipótesis) o una consecuencia lógica de otras proposiciones de la teorfa. Finalmente, una teoria cientifica es una teoria comprobable empíricamente, o al menos convertible (por especificación o agregado de premisas) en una teoría verificable mediante datos observacionales o experimentales. Ejemplo de doctrina: el estructuralismo. Ejemplo de teoría: la teoría de la utilidad (o valor subjetivo) de von Neumann y Morgenstern. Ejemplo de teoría científica: la teoría de Coleman, de las redes de influencia social.

Por ser precisa y estar organizada lógicamente, una teoría especifica permite el planteamiento y la solución de problemas bien determinados, entre ellos la predicción (o la retrodicción) de acontecimientos. Y la confrontación de tales anticipaciones con los datos empíricos, junto con el examen de la teoría a la luz de otras teorias aceptadas anteriormente, es lo que permite pronunciarse acerca del grado de verdad de la teoria. (Por ejemplo, para poner a prueba una teoría sociológica no bastan datos sociológicos: también son menester teorías económicas y psicológicas.) Si la teoría pasa tal doble examen, empírico y conceptual, se la declara teoria cientifica verdadera, al menos en algún grado y hasta nuevo aviso. Pero en ausencia de tales tests habrá que suspender el juicio sobre el valor de verdad de la teoría.

Una mera doctrina, en cambio, es menos precisa que una teoría. Por consiguiente sus predicciones, si está en condiciones de hacerlas, son imprecisas. Luego los datos empíricos no pueden decidir acerca de su valor de verdad. Esto no quita que una doctrina pueda tener valor heurístico, por ejemplo al ayudar a clasificar o a dirigir la atención hacia determinadas variables que de otro modo pasarian inadvertidas. (Este es el caso del materialismo histórico.) Pero, precisamente por no ser comprobable empíricamente, una mera doctrina no puede aspirar al rango de teoría científica verdadera, o siquiera de teoría a secas.

Algunas doctrinas pueden ser convertidas en teorias propiamente dichas y aun en teorías científicas. En cambio otras quedan forzosamente en estado gaseoso. Por ejemplo, las doctrinas de Freud y Parsons parecen ser rebeldes a tal transformación. En todo caso hasta ahora estas doctrinas no han sido convertidas en teorías científicas. Luego no puede afirmarse que sean verdaderas o falsas.

En sociología es frecuente llamar interpretación a lo que hemos denominado doctrina, acaso porque existen doctrinas, tales como el estructuralismo, el psicoanálisis y el materialismo histórico, que suelen llamarse interpreta- 
ciones. Pero en la semántica y en la epistemología contemporáneas el término "interpretación" se reserva para designar la operación consistente en asig. nar un significado determinado a una teoría abstracta (no interpretada). No obstante, dado que en las ciencias de la realidad todas las doctrinas y teorias son interpretadas (en el sentido semántico del término), no hay peligro grave de confusión: las palabras "doctrina" e "interpretación" podrán seguir usándose indistintamente. Con todo, es conveniente esforzarse por unificar las terminologías de las metodologías de las diversas ciencias de la realidad, a fin de evitar confusiones.

\section{Teoria general y teoria especifica o modelo teórico}

Una teoria general, como lo indica su nombre, concierne a todo un género de objetos, en tanto que una teoria especifica se refiere a una de las especies de tal género. Por ejemplo, una teoría de la movilidad social en general difiere de una teoría de la movilidad social en los países capitalistas, en que esta segunda teoria es más específica. En efecto, la última contiene hipótesis (p. ej. acerca de los mecanismos de la movilidad) que no están contenidas en la correspondiente teoría general.

Por cada teoría general $G$ hay entonces toda una clase de teorías especiales $E_{i}$, donde $i$ es un número natural. Cada una de estas teorías especiales $E_{i}$ contiene a la teoria general $G$ y, además, ciertas hipótesis subsidiarias $S_{i j}$ que describen las peculiaridades de la especie $i$ de objetos a que se refieren. Por cada especie $i$ habrá un conjunto de hipótesis subsidiarias: $S_{i 1}, S_{i q}$, etc. Hemos llamado $S_{i j}$ a un miembro genérico de este conjunto. $O$ sea, se tiene

$$
E_{i}=G \& S_{i 1} \& S_{i q} \& \text { \& . \& } S_{i n} \text {, }
$$

donde $n$ es el número de hipótesis subsidiarias que caracterizan a $E_{i}$ con respecto a $G$.

Se puede decir que la teoría general "abarca" a cada una de las teorías específicas correspondientes, en el sentido de que éstas se obtienen con sólo agregarle a $G$ ciertas premisas específicas. Pero es falso, aunque se lea a menudo, que $G$ contenga o implique a todas las teorías específicas $E_{i}$. Más bien es al revés: $E_{i}$ implica a $G$, esto es, lo genérico se deduce de lo específico, que es más rico. En otras palabras: dado un conjunto de teorías específicas, se puede extraer de estas una teoría general con sólo suprimir todas las premisas particulares y dejar las suposiciones comunes a todas las teorías específicas de marras.

\section{Tipo ideal}

El concepto weberiano de tipo ideal dista de ser claro. Unas veces el tipo ideal es el modelo a imitar, o norma de acción del agente racional; otras 
el tipo ideal es el modelo teórico que contiene idealizaciones extremas. En todo caso no parece que ninguno de estos dos conceptos designados por la expresión ambigua "tipo ideal" ayude mucho a comprender las idealizaciones científicas por excelencia, a saber, los modelos teóricos y, en particular, los modelos matemáticos. Todos estos contienen, desde luego, idealizaciones; toda teoría, sea específica, sea genérica, esquematiza la realidad y, más aún, constituye un objeto ideal (conceptual). Pero esto no basta para caracterizar una teoría científica: también las clasificaciones esquematizan y lo propio hacen las doctrinas no cientificas. Todas ellas son tipos ideales en la segunda acepción de este término.

Debido a la ambigüedad de la expresión "tipo ideal", que acaba de señalarse, es dudoso que convenga conservarla.

\section{Valor y objeto valioso}

Los sociólogos identifican a veces los conceptos de valor y de objeto valioso, al modo en que los grandes almacenes norteamericanos anuncian la venta de big values. En realidad hay tan sólo objetos valiosos (o disvaliosos, o desprovistos de valor), o sea, objetos a los que asignamos algún valor (en algún respecto). La noción de valor es entonces tan sólo una componente del concepto complejo objeto-al-que-asignamos-valor-en-cierto-respecto.

Lo que precede se comprende claramente si se supone que todo sujeto humano $x$ puede asignar, en principio, un determinado valor $v$ a cualquier objeto $y$. (El que esta valuación cambie con las circunstancias no se niega, sino que se puede expresar agregando una variable, por ejemplo, el tiempo.) En otras palabras, se tiene

$$
V(x, y)=v,
$$

donde $V$ es una función de valuación. Esta. representación pone en claro la diferencia entre el sujeto $x$ que efectúa la valuación, el objeto $y$ evaluado, y el valor $v$ que $x$ asigna a $y$. Cámbiese el sujeto, y el valor podrá cambiar aun cuando no cambie el objeto. Esto no quiere decir que los valores sean totalmente subjetivos, o sea, dependientes tan sólo del sujeto: dependen de ambos, del sujeto y del objeto, como lo exhibe la fórmula. Naturalmente, lo que precede no tiene sentido a menos que se especifique (a) cómo se combinan los objetos evaluados y (b) qué propiedades matemáticas tiene la función $V$. Estas tareas competen a la moderna teoría de los valores.

El esquema de axiología esbozado arriba, aunque insuficiente para analizar con profundidad el concepto de valor, basta para aclarar ciertos problemas que se presentan en las ciencias sociales. Uno de ellos es el de los conflictos de valores. Según algunos politólogos, el conflicto de valores no admite 
compromisos; y cuando falla el compromiso, sobreviene la revuelta. La afirmación es verdadera solamente en el caso extremo, por tanto infrecuente, en que se enfrentan dos finalidades o desiderata que son a la vez supremos y mutuamente incompatibles. Pero en las situaciones corrientes los agentes tienen finalidades más o menos diferentes, a las que asignan valores diferentes. Por lo tanto pueden transigir en algún respecto con tal de poder salirse con la suya en algún otro. Ejemplo: el actor a (individuo o grupo) posee las finalidades $f_{I}$ y $f_{z}$ pero le asigna a la primera un valor mucho mayor que a la segunda. $O$ sea,

$$
V\left(a, f_{2}\right)>>V\left(a, f_{2}\right)>0 .
$$

El actor $b$, en cambio, se opone a $f_{z}$, o sea, para él vale $V\left(b, f_{z}\right)<0$. Ambos pueden evitar el conflicto si a transige, al menos momentáneamente, prescindiendo de $f_{2}$. La discrepancia evaluativa subsiste pero no se manifiesta como conflicto: el actor $a$ sacrifica su finalidad $f_{\xi}$, al menos temporariamente, en aras de su finalidad más valiosa $f_{1}$. Esto ocurre diariamente en la vida de los individuos y es patente en las alianzas políticas en todo nivel.

\section{Conclusiones}

El lenguaje de toda ciencia tiene impurezas que obstaculizan la búsqueda y la transmisión del conocimiento. Esas impurezas de lenguaje son a menudo manifestaciones de oscuridades conceptuales. Y cuando de ésto se trata, el filósofo de inclinación analítica puede ser de utilidad. En algunos casos le bastará invocar la autoridad del diccionario, pero las más de las veces deberá efectuar una reconstrucción con materiales y herramientas contemporáneos. Semejante reconstrucción se impone a menudo pero no basta: la mejor manera de aclarar las ideas es sistematizarlas, es decir, incorporarlas en teorias. En el caso de las ciencias sociales, el problema es que hay pocas teorías y muchas doctrinas $u$ opiniones. También hay una notoria resistencia a la teorización, debida a una filosofía anticuada que confunde teoría científica con especulación desenfrenada. También en este punto podrá ayudar el filósofo, no sólo haciendo el elogio de la teoría, sino también ayudando a construirla o, al menos, a organizarla mejor.

Mario Bunge

INSTITUto DE INVESTigaciones Filosóficas Universidad Nacional Autónoma de MÉxuco 